\title{
Three new Eustala (Araneae, Araneidae) species from the Galápagos Islands (Ecuador)
}

\author{
Léon BAERT \\ O.D. Taxonomy and Phylogeny, Royal Belgian Institute of Natural Sciences, Vautierstraat 29, \\ B-1000, Brussels, Belgium. \\ E-mail: leon.baert@naturalsciences.be \\ urn:1sid:zoobank.org:author:05FB6C55-2E74-4AF2-86A5-83B738C6BA66
}

\begin{abstract}
Three new Eustala species are described from the Galápagos archipelago: Eustala occidentalis sp. nov. with a western distribution, Eustala orientalis sp. nov. with an eastern distribution and Eustala meridionalis sp. nov. with a distribution restricted to the southern island Floreana.
\end{abstract}

Key words. new species, colour variation, island fauna

Baert L. 2014. Three new Eustala (Araneae, Araneidae) species from the Galápagos Islands (Ecuador). European Journal of Taxonomy 86: 1-18. http://dx.doi.org/10.5852/ejt.2014.86

\section{Introduction}

Because they construct conspicuous orbwebs, Araneidae are among the most prominent group of spiders. With 169 genera and more than 3000 described species, it is also one of the largest spider families (Jocqué et al. 2013; Platnick 2014 ). On the Galápagos Islands, the family is represented by nine genera: Cyclosa Menge, 1866, Galaporella Levi, 2009, Gasteracantha Sundevall, 1833, Mastophora Holmberg, 1876, Metazygia F.O.Pickard-Cambridge, 1904, Metepeira F.O.Pickard-Cambridge, 1903 and Neoscona Simon, 1864 are represented by one species each, and Argiope Audouin, 1826 by two species (Baert et al. 2008; Baert 2013). In this paper, I report on the ninth genus, Eustala Simon, 1895, which is the most speciose araneid genus of the archipelago with three species, here described as new.

The American continent harbours 82 species of the genus Eustala, of which half are found in South America. Most of these (35 species) occur in the eastern and southern part (Guyana, Brazil, Uruguay and Argentina) of the South American mainland. Only six species are cited from the western part, more precisely from Peru, but none from Ecuador (Platnick 2014).

The Eustala specimens found on the Galápagos have so far been assigned to one species: Eustala vegeta (Keyserling, 1865) (Baert et al. 2008; Garrett et al. 2008). However, a more detailed analysis of the male palp and the female scapus shows that they belong to a complex of three different species, each with a well defined, segregated distribution over the islands of the archipelago. The males can easily be distinguished by the structure of their terminal apophyses, the females by the shape of their scapus. 


\section{Material and methods}

The material studied consists of samples taken by the following sampling teams (field numbers in brackets): L. Baert \& J.-P. Maelfait (82/LB,JPM), L. Baert, J.-P. Maelfait \& K. Desender (86/ LB,JPM,KD; 88/LB,JPM,KD; 91/LB,JPM,KD; 00/LB,JPM,KD), J. Herathy (91/JH), S. Peck (89/SP; 92/SP; 96/SP), W.G. Reeder (75/WGR; 77/WGR; 78/WGR; 79/WGR; 80/WGR), S. Riechert (70/SR) and L. Roque (99/LR).

The type material of the three species is deposited in the collections of the Royal Belgian Institute of Natural Sciences, Brussels (Belgium).

Specimens were examined and measured with a Wild M5 stereo-microscope, and illustrated using a Wild M10 stereomicroscope. The electron microscope photographs were made with an ESEM FEI QUANTA 200 microscope. The colour photographs were made with a Nikon D7000 with an AF Micro Nikkor 60 $\mathrm{mm}$ objective. All measurements are in $\mathrm{mm}$.

The left male palps are depicted, except the right one for E. orientalis. The female genitalia were cleared in a methylsalicylate solution.

$\begin{array}{ll}\text { Abbreviations } \\ \mathrm{AME} & =\text { Anterior Median Eyes } \\ \mathrm{Cl} & =\text { Clypeus length } \\ \mathrm{CDRS} & =\text { Charles Darwin Research Station } \\ \mathrm{DAME} & =\text { Diameter of Anterior Median Eye } \\ \mathrm{Fe} & =\text { Femur } \\ \mathrm{j}(\mathrm{s}) & =\text { juvenile(s) } \\ \mathrm{L} / \mathrm{D} & =\text { length of scapus/diameter of scapus } \\ \mathrm{MOQ} & =\text { Median Ocular Quadrat } \\ \mathrm{Mt} & =\text { Metatarsus } \\ \mathrm{Pa} & =\text { Patella } \\ \mathrm{PLE} & =\text { Posterior Lateral Eye } \\ \mathrm{PME} & =\text { Posterior Median Eye } \\ \mathrm{SA} & =\text { Subadult } \\ \mathrm{Ta} & =\text { Tarsus } \\ \mathrm{Ti} & =\text { Tibia } \\ (\text { đ) } & =\text { subadult male } \\ (+) & =\text { subadult female }\end{array}$

\section{Results}

Taxonomy

Class Arachnida Cuvier, 1812

Order Araneae Clerck, 1757

Family Araneidae Clerck, 1757

Genus Eustala Simon, 1895

\section{Diagnosis of the genus}

The carapace has a deep longitudinal cleft in the thoracic region. The abdomen has a triangular shape with a dorsal folium pattern and a ventral white patch. The posterior median eyes are usually slightly smaller than the anterior median eyes. The males are smaller than the females. The distal margin of the 
BAERT L., Three new Eustala (Araneae) species from the Galápagos Island

male first coxa has a distinct hook, which fits into a groove on the second femur. The palpal patella has one very long macroseta. The bulb has a huge, variably-shaped conductor and a conspicuous white, cone-shaped median apophysis produced downward on the ventral side of the large bulb. Females are characterized by the scapus of the epigyne projecting forward.

The males of the three species described here differ from all known Eustala species by the structure of the terminal apophysis, the subterminal apophysis and the embolus, the females by the shape of the anteriorly projecting scapus.

The males of these three Galápagos species differ from each other by the structure of their terminal apophysis (Fig. 6) while the females are recognized by the shape of their scapus (Figs 1C, 3C, 4C).

Eustala occidentalis sp. nov.

urn:1sid:zoobank.org:act:95A5F993-4A60-4567-ABAD-9D8489A5EDD3

Figs 1 A-E, 2 A-F, 5 A, 6 A, 7

Epeira prompta Hentz, 1847. - Banks 1902: 61; 1924: 97. — Snodgrass 1902: 76. — Roth \& Craig 1970: 120 (misidentification).

Eustala sp. - Roth \& Craig 1970: 116. — Baert 2013: 176, 178

Eustala vegeta (Keyserling, 1865). - Baert, Maelfait, Hendrickx \& Desender 2008: p. 47, Map 15 Garrett, Conner \& Roque-Albelo 2008: 2-4, f. 1 (misidentification).

\section{Diagnosis}

Male: Terminal apophysis needle-like, short and slightly curved, its apical and caudal ridges equal in length and structure.

\section{Etymology}

The species name refers to its distribution in the western part of the archipelago.

\section{Type material}

\section{Holotype}

đ, ISLA SANTA CRUZ, Bahía Tortuga, arid zone, 14 Mar. 1986, leg. Baert, Maelfait \& Desender.

\section{Allotype}

, ISLA SANTA CRUZ, Along the road connecting the Charles Darwin Research Station to Puerto Ayora, Arid zone, 15 Apr. 1982, leg. Baert \& Maelfait.

\section{Paratypes}

ISLA SANTA CRUZ: 1 đ, 1 †, CDRS, 1 Mar. 1986 (B.86/046); 1 §, CDRS, 2 Mar. 1986 (B.86/047);

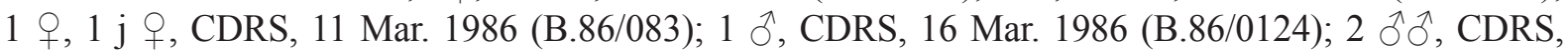
dormitorio, Mar. 1986 (leg. Baert, Maelfait \& Desender 1986). 1 §ૈ, 2 j, CDRS, dormitorio, 17 Feb. 1988 (B.88/317) (leg. Baert, Maelfait \& Desender 1988); 1 §, 3 km South of Bellavista, alt. 115 m, 1-29 Feb. 1989 (P.89/81); 1 ô, CDRS, dormitorio, Apr. 1991 (B.91/757) (leg. Baert, Maelfait \& Desender, 1991); 1 †, 2.7 km of Los Gemelos, alt. 500 m, 23 Mar. 1996 (B.96/6); 1 क, Media Luna, alt. 600m, 11 Mar. 1998 (B.98/015) (leg. Baert, Maelfait \& Desender, 1998); 1 , 1 (ㅇ), 1 (ङ ), 2 j(s), Cerro Colourado, alt. $10 \mathrm{~m}$, 10 Mar. 1975 (leg. W.G. Reeder); 1 +, Horneman farm, Bellavista, alt. 225 m, cultivated zone, 14 Oct. 1975 (leg. W.G. Reeder); 1 क , 1 j, nesting zone 3 area, 1 Aug. 1970 (leg. Riechert); 1 q, northern base of Media Luna, alt. 610-620 m, open shrub savanna of Miconia surrounded by ferns and grasses, 3 May 1980 (leg. W.G. Reeder); 2 우, 1 ( §), 1 (), 3 j(s), CDRS, Academy Bay, altitude 5-15 m, from Scutia, Croton, Castela and Cryptocarpus plants, 16 Aug. 1977 (leg. W.G. Reeder); 1 q, 6 j(s), CDRS, Academy Bay, alt. 10 m, from Scutia, Cryptocarpus and Scalesia plants, 18 Oct. 1975 (leg. W.G. Reeder). 

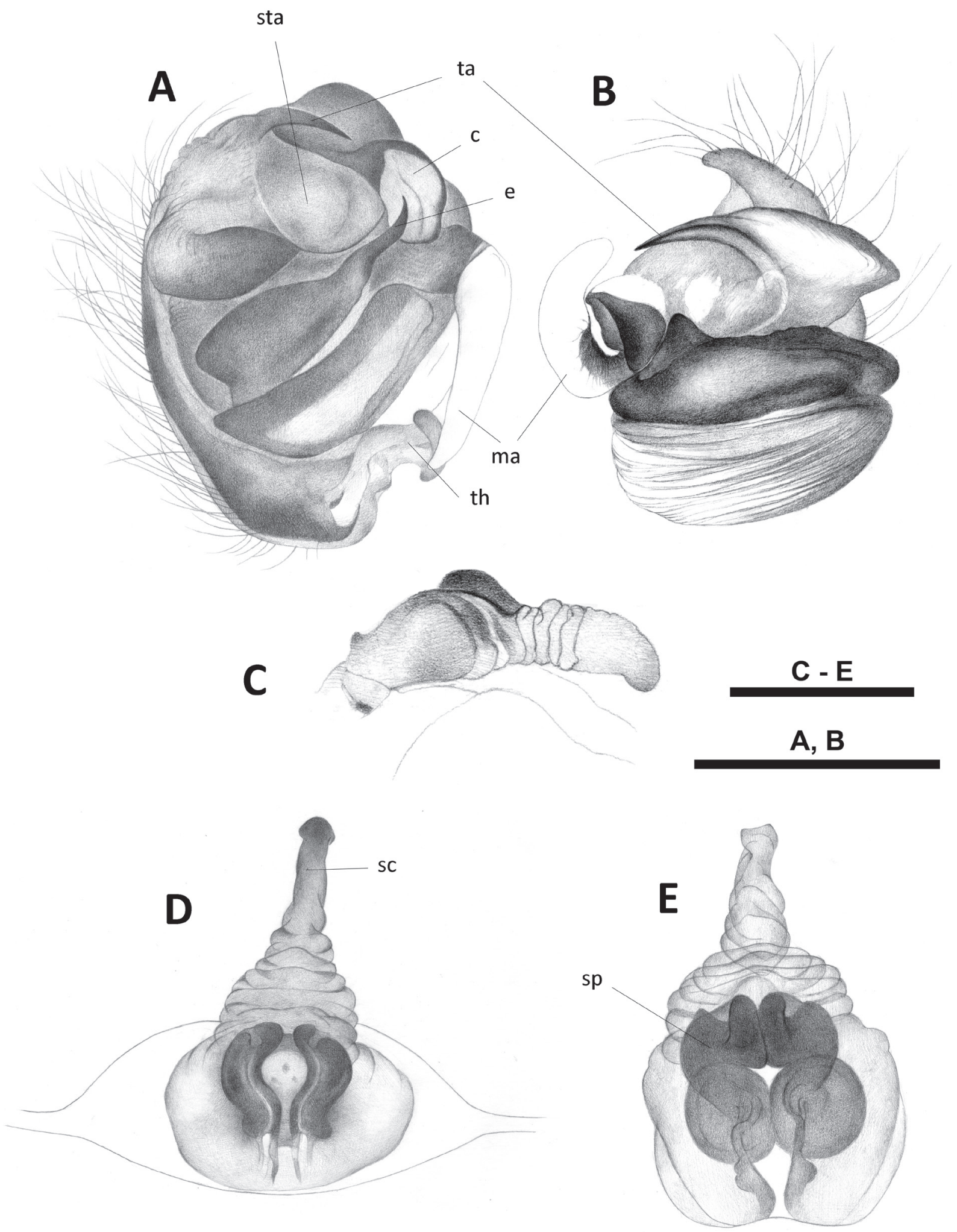

Fig. 1. Eustala occidentalis sp. nov. A. $\precsim$ left palp, ventral view. B. $ð$ left palp, apical view. C. + scapus, lateral view. D. $q$ epigyne scapus, ventral view. $\mathbf{E}$. + , spermathecae. $\mathrm{c}=$ conductor, $\mathrm{e}=$ embolus, $\mathrm{ma}=$ median apophysis, $\mathrm{sc}=$ scapus, $\mathrm{sp}=$ spermathecae, $\mathrm{sta}=$ subterminal apophysis, ta $:$ terminal apophysis, th $=$ tarsal hook. Scale lines $=0.5 \mathrm{~mm}$. 
BAERT L., Three new Eustala (Araneae) species from the Galápagos Island

RBINS inventory number of all type material is I.G. 32722.

Other material examined originating from the islands Fernandina, Isabela (Volcán Sierra Negra, Volcán Alcedo, Volcán Darwin), Marchena, Pinta and Santiago (see Table 1).

\section{Description}

\section{Male (holotype)}

LENGTH. Total length 5.80; carapace length 3.14, width 2.63, height 0.75 ; abdomen length 3.65 , width 2.63 .

CARAPACE. Colour (in alcohol) yellowish with clypeus and eye region black, dorsal cephalic part black with central yellow V; two short, diverging combs of long, thin, white hairs starting from PME and PLE. Chelicerae black with few yellow stains. Labium black with white apical edge. Endites black with white apical and inner edges. Sternum white with irregular blackish suffused border. Pedipalps with striped yellow-black appearance. Legs yellow with variable black annulations.

АвDomen. Dorsum white with distinct dorsal dark folium pattern (colour pattern A), with six sclerotized apodemes; venter black with central white patch; spinnerets dark.

EYES. MOQ: PME $=0.72$ AME. $\mathrm{Cl}=0.5$ DAME.

Legs. Measurements:

I (15.23): Fe 4.63, Pa 1.65, Ti 3.73, Mt 3.73, Ta 1.49

II (11.48): Fe 3.57, Pa 1.25, Ti 2.43, Mt 2.94, Ta 1.29

III (6.81): Fe 2.43, Pa 0.86, Ti 1.33, Mt 1.37, Ta 0.82

IV (10.35): Fe 3.57, Pa 1.25, Ti 2.20, Mt 2.39, Ta 0.94

Sination. All legs with numerous spines.

Pedipalp (Figs 1A-B, 5A). One very long patellar seta, slightly longer than bulbus. Cymbium with conspicious T-shaped tarsal hook, its transverse bar slightly curved and with rounded extremities. Terminal apophysis (Fig. 6 A) needle-like, slightly bent, short, apical and caudal ridges equal in shape and length; embolus short, sharp tip, slightly curved at tip, lying in excavation of small, whitish conductor.

\section{Female (allotype)}

LENGTH. Total length 10.72; carapace length 4.20 , width 3.53 , height 1.18 ; abdomen length 7.35 , width 6.78 .

CARAPACE. Colour: orange brown; chelicerae creamy orange; labium, endites and sternum orange brown. Legs orange brown.

ABDomen. Creamy white, faintly speckled with cream spots, four apodemes; grey patches extend laterally of the proximal dots; three branched, dirty-grey lines run backwards in distal half of dorsum; venter cream with central white patch; spinnerets orange brown.

EYES. MOQ: $\mathrm{PME}=0.79$ AME. $\mathrm{Cl}=0.9$ DAME.

LEGS. Measurements:

I (18.21): Fe 5.47, Pa 2.27, Ti 4.31, Mt 4.47, Ta 1.69

II (15.41): Fe 4.59, Pa 2.12, Ti 3.45, Mt 3.80, Ta 1.45

III (9.29): Fe 3.14, Pa 1.37, Ti 1.88, Mt 1.92, Ta 0.98

IV (14.28): Fe 4.98, Pa 1.96, Ti 3.06, Mt 3.18, Ta 1.10 
Table 1. Other material examined of Eustala occidentalis sp. nov. Abbreviations: BAL: Baltra, EDE: Eden, FER: Fernandina, IBC: Isabela, Beagle Crater, ISN: Isabela, Sierra Negra, IVA: Isabela, Volcán Alcedo, IVD: Isabela, Volcán Darwin, MAR: Marchena, PIN: Pinta, PIZ: Pinzón, RAB: Rábida, SAN: Santiago, SEY: Seymour Norte, SPL: South Plaza; $\overbrace{}^{\Uparrow}:$ male, $\left({ }^{\Uparrow}\right)$ : subadult male, ${ }_{+}$: female, $(+)$: subadult female, j(s): juvenile(s), \#: several.

\begin{tabular}{|c|c|c|c|c|c|}
\hline Island & Locality & Altitude & Date & Sex & Lit. / Leg. \\
\hline BAL & & & & & Roth \& Craig, 1970 \\
\hline EDE & & & & j & 99/LR \\
\hline FER & $500 \mathrm{~m} \mathrm{~S}$ of Cabo Hammond & $10 \mathrm{~m}$ & 1 May 1975 & 1 우 & 75/WGR \\
\hline FER & West vegetation strip, camp area & $20 \mathrm{~m}$ & 10 Aug. 1977 & $1+1\left({ }^{\Uparrow}\right) 7 \mathrm{j}(\mathrm{s})$ & 77/WGR \\
\hline FER & Between Cabo Hammond \& Bursera Hills & $70 \mathrm{~m}$ & 26 Apr. 1975 & 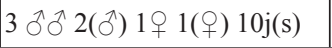 & 75/WGR \\
\hline FER & Cerro Verde & $170 \mathrm{~m}$ & 9 May 1991 & 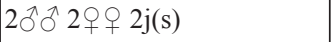 & 91/LB,JPM,KD \\
\hline FER & W slope, $500 \mathrm{~m} \mathrm{~S}$ of camp crater & $380 \mathrm{~m}$ & 16 Aug. 1977 & 1 우 & 77/WGR \\
\hline FER & W slope of camp crater & $450 \mathrm{~m}$ & 16 Aug. 1977 & 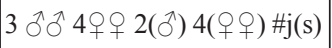 & 77/WGR \\
\hline FER & Along encañada & $600 \mathrm{~m}$ & 7 May 1991 & $1 \mathrm{j}$ 우 & 91/LB,JPM,KD \\
\hline IBC & Camp & & 22 May 1980 & $7 \lesssim 3(ふ)$ 13우 3(†) \#j(s) & 80/WGR \\
\hline IBC & Outside crater wall & $40-50 \mathrm{~m}$ & 22 May 1980 & $1 \delta^{\lambda} 2 \mathrm{j}(\mathrm{s})$ & 80/WGR \\
\hline ISN & Lagoons of Villamil & & 14 Jul. 1970 & 1 우 & 70/SR \\
\hline ISN & Near Villamil (road to highlands, $1 \mathrm{~km}$ from sea) & $10-15 \mathrm{~m}$ & 11 Jul. 1978 & $2 \overbrace{}^{\lambda} 2$ 웅 & 78/WGR \\
\hline ISN & Camp above Alemania & $540 \mathrm{~m}$ & 24 Jan. 1975 & 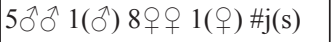 & 75/WGR \\
\hline ISN & Camp above Alemania & $540 \mathrm{~m}$ & 23 Jan. 1978 & $10^{\lambda} 5$ 우우 & 78/WGR \\
\hline ISN & Crater rim & $925 \mathrm{~m}$ & 19 Feb. 1986 & $1 \hat{O}^{\lambda}$ & 86/LB,JPM,KD \\
\hline IVA & E slope & $340 \mathrm{~m}$ & 18 May 1980 & $10^{\lambda}$ & 80/WGR \\
\hline IVA & West of lava flow (W slope) & $370-380 \mathrm{~m}$ & 25 May 1980 & $2 \hat{\jmath} 8$ 8우 \#j(s) & 80/WGR \\
\hline IVA & Pega Pega camp & $380 \mathrm{~m}$ & 23 May 1980 & $1 \overbrace{}^{\lambda} 2\left({ }^{\lambda}\right) 4(+)$ 6j(s) & 80/WGR \\
\hline IVA & E slope & $380 \mathrm{~m}$ & 25 May 1980 & $10^{\pi}$ & 80/WGR \\
\hline IVA & W slope & $620-1260 \mathrm{~m}$ & 21-23 May 1980 & 1 우우 & 80/WGR \\
\hline IVA & Pega Pega camp & $780 \mathrm{~m}$ & 13 May 1980 & 107 우우 \#j(s) & 80/WGR \\
\hline IVA & West of lava flow (W slope) & & 23 May 1980 & $191(\%) 2 \mathrm{j}(\mathrm{s})$ & 80/WGR \\
\hline IVA & East of lava flow & & 24 May 1980 & $1+6 \mathrm{j}(\mathrm{s})$ & 80/WGR \\
\hline IVA & East rim & $1045 \mathrm{~m}$ & 16 May 1980 & 1 우 & 80/WGR \\
\hline IVA & East rim & $1045 \mathrm{~m}$ & 17 May 1980 & $1 \lesssim 7$ 우우 \#j(s) & 80/WGR \\
\hline IVD & Tagus Cove & $20 \mathrm{~m}$ & 13-22 May 1992 & $10^{\pi}$ & 92/SP \\
\hline IVD & Flat between IBC \& IVD & $40-50 \mathrm{~m}$ & 22 May 1980 & 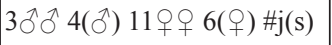 & 80/WGR \\
\hline MAR & Playa Negra & & 10 Mar. 1988 & $\# \mathrm{j}(\mathrm{s})$ & 88/LB,JPM,KD \\
\hline MAR & Punto Mejio & & 11 Mar. 1988 & $1 \mathrm{j}$ 우 & 88/LB,JPM,KD \\
\hline MAR & Playa Negra & & 29 Mar. 2000 & $20 \hat{\jmath} 1$ 19 & 00/LB,JPM,KD \\
\hline MAR & SW slope of beach camp area & $5 \mathrm{~m}$ & 24 Jan. 1977 & $1{ }^{\Uparrow} 4$ 웅 3(우) 7j(s) & 77/WGR \\
\hline MAR & Above beach at Beach camp (S slope) & $5-10 \mathrm{~m}$ & 26 Jan. 1977 & 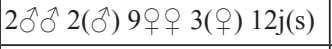 & 77/WGR \\
\hline MAR & S Playa camp, new lava flow & $16-20 \mathrm{~m}$ & 31 Jan. 1977 & $1 \circlearrowleft 1(ð) 2 \mathrm{j}(\mathrm{s})$ & 77/WGR \\
\hline MAR & SW Playa, Bursera forest & $30 \mathrm{~m}$ & 12-24 Mar. 1992 & $10^{\lambda}$ & 92/SP \\
\hline MAR & & $50 \mathrm{~m}$ & 10 Mar. 1988 & $1 \mathrm{j}$ 우 & 88/LB,JPM,KD \\
\hline MAR & Fumarole at Barranco & $160-190 \mathrm{~m}$ & 29 Jan. 1977 & $2 \widehat{\jmath} 2+q$ 3(ㅇ) $5 \mathrm{j}(\mathrm{s})$ & 77/WGR \\
\hline PIN & Cabo Ibbetson & & 31 Mar. 2000 & $10^{\lambda}$ & 00/LB,JPM,KD \\
\hline
\end{tabular}


BAERT L., Three new Eustala (Araneae) species from the Galápagos Island

\begin{tabular}{|c|c|c|c|c|c|}
\hline Island & Locality & Altitude & Date & Sex & Leg. \\
\hline PIN & S slope, camp area & $250 \mathrm{~m}$ & 19 Jul. 1975 & $1 \mathrm{~m} 3$ 우우 $1(\mathrm{~m}) \mathrm{\# j}(\mathrm{s})$ & 75/WGR \\
\hline PIN & S slope, camp area & $250 \mathrm{~m}$ & 20 Jan. 1977 & $2 q$ & 77/WGR \\
\hline PIN & E slope & $300 \mathrm{~m}$ & 20 Mar. 1986 & 1 우 & 86/LB,JPM,KD \\
\hline PIN & W slope & $300 \mathrm{~m}$ & 21 Mar. 1986 & $10^{\lambda}$ & 86/LB,JPM,KD \\
\hline PIN & E slope & $360 \mathrm{~m}$ & 19 Mar. 1986 & $10^{\lambda}$ & 86/LB,JPM,KD \\
\hline PIN & W slope & $400 \mathrm{~m}$ & 30 Mar. 2000 & 1 우 & 00/LB,JPM,KD \\
\hline PIN & Below summit & $630 \mathrm{~m}$ & 22 Jul. 1977 & 1 우 & 77/WGR \\
\hline PIZ & Old crater camp & $300-320 \mathrm{~m}$ & 5 Feb. 1979 & $2\left(O^{\pi}\right) \# \mathrm{j}(\mathrm{s})$ & 79/WGR \\
\hline PIZ & Crater camp ridge summit & $320 \mathrm{~m}$ & 6 Feb. 1979 & 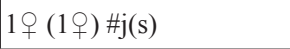 & 79/WGR \\
\hline RAB & Margins of lagoon & $3-10 \mathrm{~m}$ & 28 Sep. 1975 & $1{ }^{\Uparrow} 3 \mathrm{j}(\mathrm{s})$ 우 & 75/WGR \\
\hline SAN & $7 \mathrm{~km} \mathrm{SE}$ of Playa Espumila & & 9 Jun. 1991 & $1 \mathrm{j}$ 우 & 91/JH \\
\hline SAN & Bahía Bucanero (behind beach) & & 14 Apr. 1975 & $195 \mathrm{j}(\mathrm{s})$ & 75/WGR \\
\hline SAN & Bahía Bucanero & $3 \mathrm{~m}$ & 18 Sep. 1975 & 2 우 1(우) & 75/WGR \\
\hline SAN & Bahía Bucanero & $5-10 \mathrm{~m}$ & 10 Sep. 1975 & 1 우 & 75/WGR \\
\hline SAN & Bahía Bucanero & $10-15 \mathrm{~m}$ & 12 Sep. 1975 & 1 iq & 75/WGR \\
\hline SAN & Bahía Bucanero & $15 \mathrm{~m}$ & 17 Apr. 1975 & $1 \mathrm{j}$ ㅇ $2 \mathrm{j}(\mathrm{s})$ & 75/WGR \\
\hline SAN & Mina de sal & $50 \mathrm{~m}$ & 8 Mar. 1986 & 1 iq & 86/LB,JPM,KD \\
\hline SAN & Transect from Bahía Bucanero to summit & $75 \mathrm{~m}$ & 11 Sep. 1975 & 1 우 & 75/WGR \\
\hline SAN & Cerro Cowan & $260 \mathrm{~m}$ & 7 Apr. 1982 & 1 iq & 82/LB,JPM \\
\hline SAN & N slope & $400 \mathrm{~m}$ & 4 Mar. 1986 & $1(+)$ & 86/LB,JPM,KD \\
\hline SAN & SW slope & $400 \mathrm{~m}$ & 7 Mar. 1986 & 19 & 86/LB,JPM,KD \\
\hline SAN & SW facing slope of crater area & $810-830 \mathrm{~m}$ & 1 Sep. 1975 & 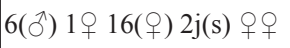 & 75/WGR \\
\hline SAN & $1.5 \mathrm{~km} \mathrm{~S}$ of summit (tree fern valley) & $875 \mathrm{~m}$ & 21 Sep. 1975 & $4 \mathrm{j}(\mathrm{s})$ & 75/WGR \\
\hline SEY & & $10 \mathrm{~m}$ & 26 Oct. 1975 & 1 + & 75/WGR \\
\hline SPL & & $10-15 \mathrm{~m}$ & 19 Mar. 1975 & $3 \widehat{\partial} \widehat{\partial} 1$ 우 $2 \mathrm{j}(\mathrm{s})$ & 75/WGR \\
\hline
\end{tabular}

Epigyne (Fig. 1C-E). Length of scapus 0.91, diameter (lateral view) 0.19, L/D = 2.95. Spermathecae touching each other medially; scapus evenly broad (ventral view), broadest in the middle, tip slightly curved (lateral view).

\section{Variation}

The males in our samples vary in length between 4.9 and $7.4 \mathrm{~mm}$, the females between 5.7 and $10.6 \mathrm{~mm}$.

A great diversity in abdominal colouration, independent of island origin, can be observed (Figure 2). The abdominal colour can vary from uniform light cream (Pattern A: for example $\overbrace{}^{\lambda}$ of sample B.88/317: Santa Cruz, surroundings of CDRS, 17 Feb. 1988; Allotype $q$ from Santa Cruz and $q$ from Marchena, 26/I/1977, leg. Reeder), to very dark (Pattern B: for example $q$ specimen from Santa Cruz, Media Luna, $620 \mathrm{~m}$ alt., 3 May 1980, leg. Reeder), with all possible variations in between. The colour may be uniform or with a faint or a very conspicuous folium (patterns on Fig. 2A, 2D-F), showing two sinuous lines converging backwards. Some specimens, male and females, have a black abdomen with two large white shoulder spots (pattern, Fig. 2C: for example $\widehat{\delta}$ specimen from sample P.92/179: Tagus cove, Volcán Darwin, 22 May 1992, but also some specimens from Santa Cruz, Volcán Sierra Negra, Pinta and Santiago), a few with a dorsal longitudinal white band (Fig. 2D, F) and some with a white abdomen with median black folium (Fig. 2E). Cephalothorax also variable, with or without marked black stains. 

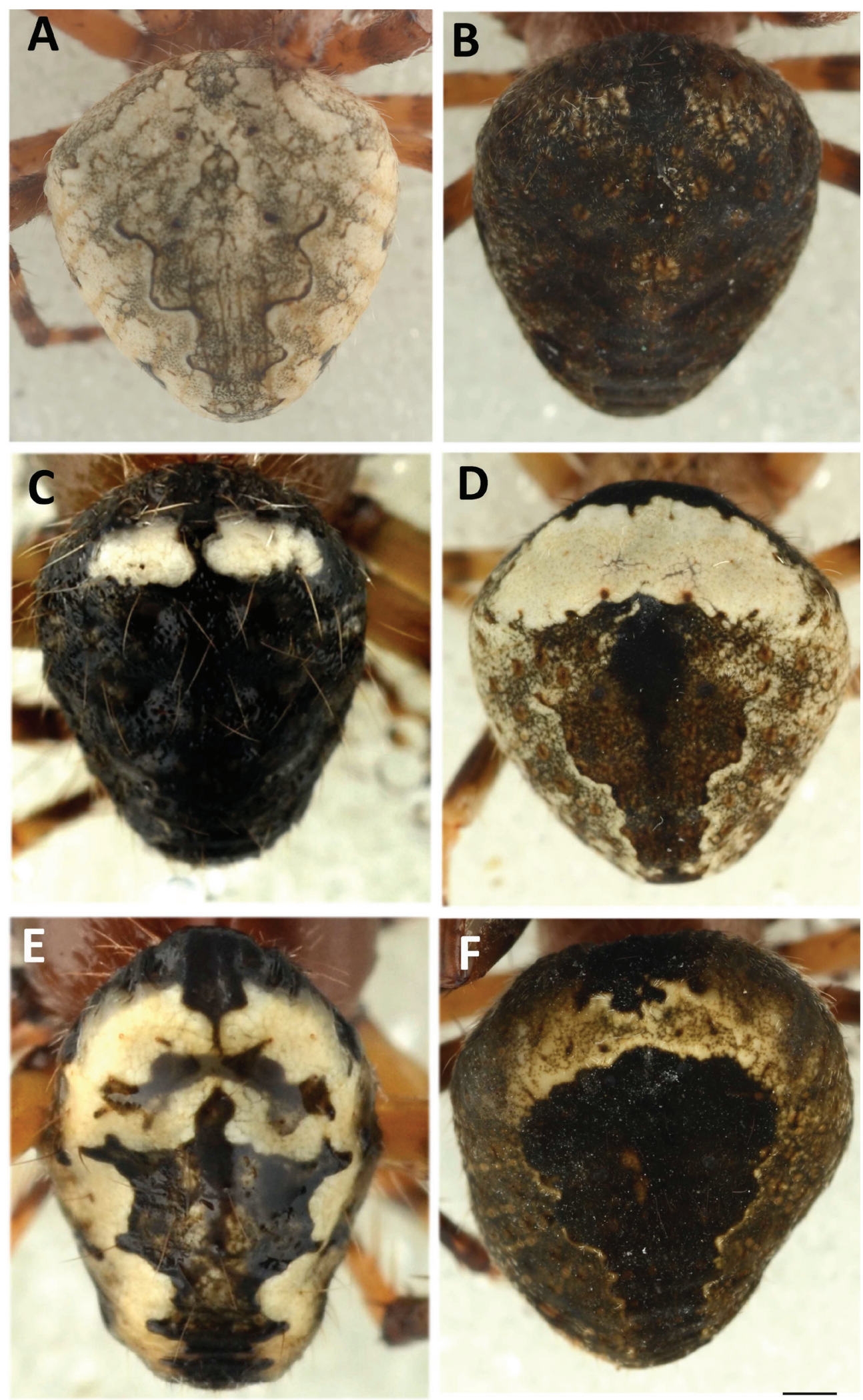

Fig. 2. Eustala occidentalis sp. nov., examples of abdominal patterns. A. $q$ from Isla Rábida. B. $q$ from Isla Santa Cruz. C. $\widehat{\partial}$ from Isla Isabela, Tagus Cove. D. $\varnothing$ from Isla Pinta. E. $\widehat{\partial}$ from Isla Santa Cruz. F. $q$ from Isla Santiago. Scale lines $=1 \mathrm{~mm}$. Photographs: Alain Pauly. 
BAERT L., Three new Eustala (Araneae) species from the Galápagos Island

\section{Distribution}

E. occidentalis sp. nov. has a western distribution within the archipelago (Fig. 7): Fernandina (up to $600 \mathrm{~m}$ alt.); the Isabela volcanoes Volcán Sierra Negra (arid zone and above $900 \mathrm{~m}$ alt.), Volcán Alcedo (above 300 m alt.), Volcán Darwin and Beagle Crater (coastal arid zone); Marchena; Pinta (from coast to summit); Pinzon (above $300 \mathrm{~m}$ alt.); Rábida; Santiago (from coast to summit); Seymour Norte; South Plaza and Santa Cruz (from coast up to $600 \mathrm{~m}$ alt.). There is apparently no preferred vegetation zone. It also occurs along the walls of buildings (cf. CDRS buildings of the dormitorio).

Eustala orientalis sp. nov. urn:1sid:zoobank.org:act:AF420B6B-A770-4731-908B-4ECF219861A9

Figs 3 A-E, 5 C, 6 C, 7

Eustala vegeta (Keyserling, 1865). - Baert, Maelfait, Hendrickx \& Desender 2008: 47, map 15 (misidentification).

Eustala sp. - Baert 2013: 176, 178.

\section{Diagnosis}

Males differ from E. occidentalis sp. nov. by the structure of the terminal apophysis: flat and broadening towards the tip, its caudal ridge being longer than the apical ridge. Females differ from E. occidentalis sp. nov. by the shape of the scapus.

\section{Etymology}

The species name refers to its distribution in the eastern part of the archipelago.

\section{Type material}

\section{Holotype}

${ }^{7}$, ISLA ESPAÑOLA, $1 \mathrm{~km} \mathrm{~W}$ of Punta Cevalos beach, vegetation of beach berm: Valessia, Grabowskia, Prosopis, Cordia, 7 Feb. 1977, leg. W.G. Reeder.

\section{Allotype}

, ISLA ESPAÑOLA, $1 \mathrm{~km} \mathrm{~W}$ of Punta Cevalos beach, vegetation of beach berm: Valessia, Grabowskia, Prosopis, Cordia, 7 Feb. 1977, leg. W.G. Reeder.

\section{Paratypes}

ISLA ESPAÑOLA: 1 đ̊, Bahía Gardner, Playa blanca, 27 Apr. 1991 (B.91/0742) (leg. Baert, Maelfait \& Desender, 1991); 1 ㅇ, 2 (우), Caleta at Bahía Manzanilla, 21 Mar. 2009 (B.09/014-15); 2 ふૈ $\widehat{0}, 6$ 우, $3(\widehat{\jmath}), \# \mathrm{j}(\mathrm{s}), 1 \mathrm{~km}$ W of Punta Cevalos beach, vegetation of upper beach: Valessia, Grabowskia, Prosopis, Cordia, 7 Feb. 1977 (leg. W.G. Reeder); 1 J, Punta Suarez, albatros colony, 28 Feb. 1977 (leg. W.G. Reeder).

ISLOTE GARDNER near ESPAÑOLA: 2 우, alt. 10-30 m, through Bursera, Croton, Alternanthera, and ferns, 12 Feb. 1977 (leg. W.G. Reeder); 1 o, alt. 30 m, from Croton in Bursera-Opuntia-CordiaCroton community, 12 Feb. 1977 (leg. W.G. Reeder).

RBINS inventory number of all type material is I.G. 32724.

Other material examined from the islands of Genovesa, San Cristóbal and Santa Fé (see Table 2). 
Table 2. Other material examined of Eustala orientalis sp. nov. Abbreviations: GEN: Genovesa, SCB:

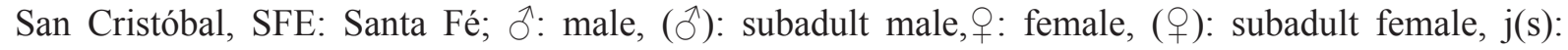
juvenile(s).

\begin{tabular}{|c|c|c|c|c|c|}
\hline Island & Locality & Altitude & Date & Sex & Leg. \\
\hline GEN & $500 \mathrm{~m} \mathrm{~W}$ of Playa Bahía Darwin & $10-15 \mathrm{~m}$ & 23 Oct. 1975 & $3++$ 1(ð) 4(ㅇ) 4j(s) & 75/WGR \\
\hline GEN & $500 \mathrm{~m} \mathrm{SE}$ of crater rim & $50 \mathrm{~m}$ & 25 Oct. 1975 & $1 \mathrm{j}$ 우 & 75/WGR \\
\hline GEN & Lago Arcturus & $60 \mathrm{~m}$ & 13 Mar. 1988 & $1 \hat{\jmath}$ & 88/LB,JPM,KD \\
\hline $\mathrm{SCB}$ & Wreck Bay area, upper beach vegetation & $5 \mathrm{~m}$ & 9 Feb. 1975 & $1 \delta^{\lambda} 19$ & 75/WGR \\
\hline $\mathrm{SCB}$ & Cerro Mundo & $550 \mathrm{~m}$ & 13-23 Feb. 1989 & $1 \delta^{\lambda}$ & 89/SP \\
\hline SCB & El Junco (near Cabo Guido Rosillo) & $600 \mathrm{~m}$ & 18 Feb. 1978 & $3 \widehat{o} 1+2 \mathrm{j}(\mathrm{s})$ & 78/WGR \\
\hline SFE & Camp area & $5-10 \mathrm{~m}$ & 24 Jan. 1979 & $2 \hat{\jmath}$ & 79/WGR \\
\hline SFE & & $\begin{array}{c}50-100 \\
\mathrm{~m}\end{array}$ & 1-2 Apr. 1982 & 1 우 & 82/LB,JPM \\
\hline SFE & $1 \mathrm{~km} \mathrm{SSW}$ of camp beach, second arranco & $100 \mathrm{~m}$ & 24 Jan. 1979 & $1 \mathrm{j}$ & 79/WGR \\
\hline
\end{tabular}

\section{Description}

\section{Male (holotype)}

LENGTH. Total length 5.06; carapace length 2.67, width 2.16, height 0.90 ; abdomen length 3.41 , width 2.27, height 1.96 .

CARAPACE. Colour (in alcohol) yellow brown with clypeus and eye region black, dorsal cephalic part yellow brown with central, faintly suffused, blackish V; two short combs of long white hairs diverge from between PME and PLE. Chelicerae yellow with central, faintly suffused, blackish stain. Labium and endites pale yellow with white margin. Sternum yellow with faintly suffused, blackish stains in front of coxae. Pedipalps yellow-brown with some black stain. Legs pale yellow with black annulations, variable between specimens; coxae and proximal part of femora whitish.

ABDomen. Dorsum creamy white with small, black median triangle in front, two small, white central stains and distinct dark sepia folium with dark brown margins and ten brown apodemes arranged as 4-2-2-2, becoming smaller caudally and towards the sides; venter whitish, slightly suffused with black; colulus and spinnerets dark.

Eyes. MOQ: PME $=0.8$ AME. $\mathrm{Cl}=0.5$ DAME.

LEGS. Measurements:

I (13.09): Fe 4.12, Pa 1.37, Ti 3.25, Mt 3.10, Ta 1.25

II (9.97): Fe 3.06, Pa 1.14, Ti 2.12, Mt 2.55, Ta 1.10

III (5.89): Fe 2.12, Pa 0.78, Ti 1.14, Mt 1.14, Ta 0.71

IV (8.94): Fe 3.10, Pa 1.10, Ti 1.88, Mt 2.04, Ta 0.82

Spination. All legs with numerous spines.

Pedipalp (Figs 3A-B, 5C). One very long patellar seta, slightly longer than bulbus. Cymbium with conspicuous, rounded, T-shaped tarsal hook. Terminal apophysis (Fig. 6C) short, caudal ridge being longer than apical ridge, flat and broadening towards tip.

Female (allotype)

LENGTH. Total length 7.10; carapace length 3.10, width 2.67, height 1.06; abdomen length 4.47, width 4.16, height 3.14. 


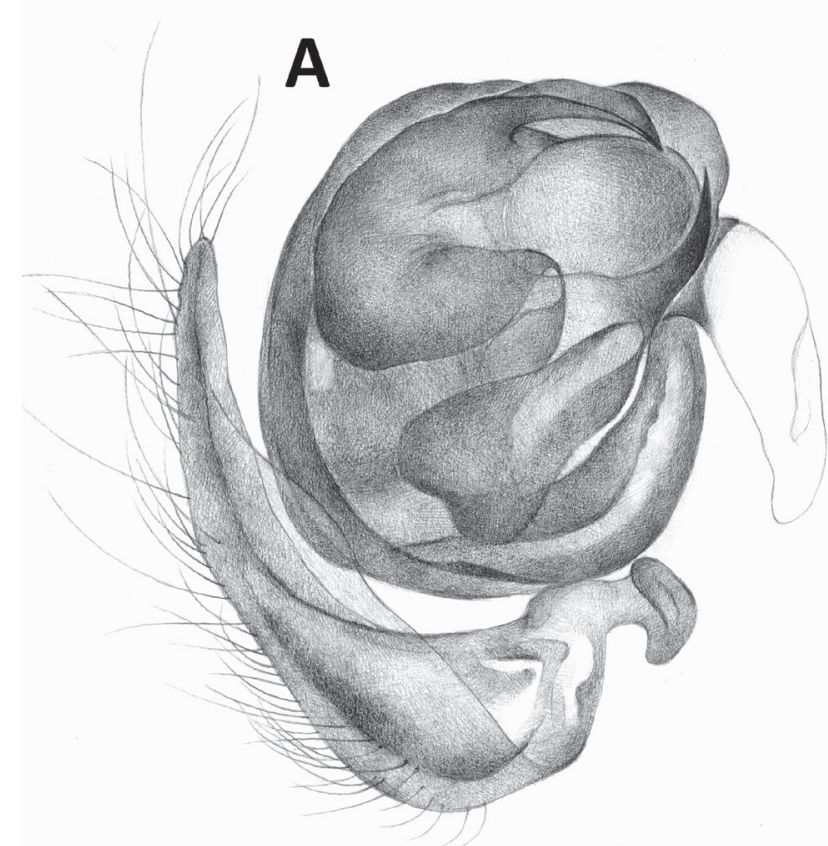

B
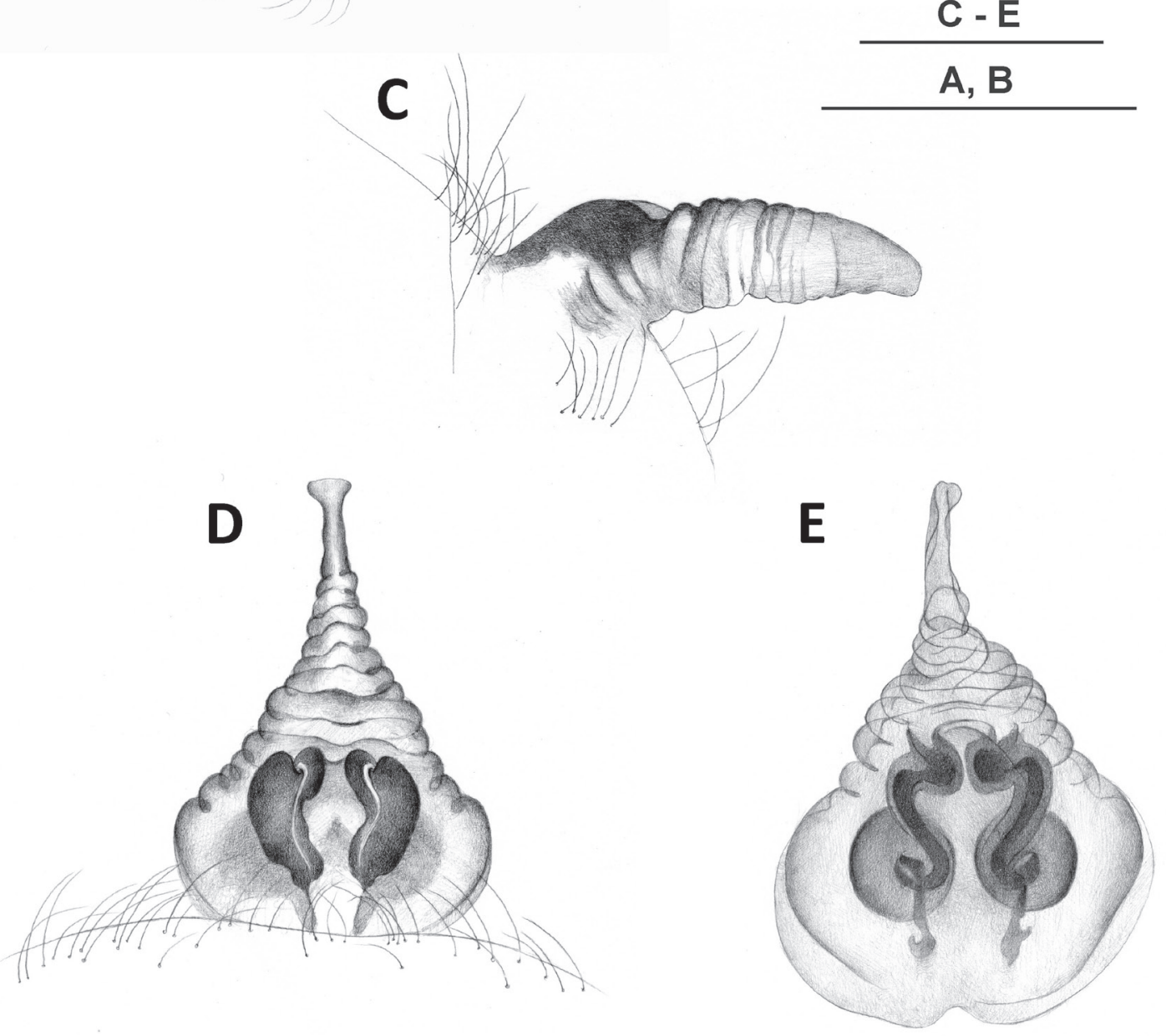

Fig. 3. Eustala orientalis sp. nov. A. $\curvearrowright$ left palp, ventral view. B. $\curvearrowright$ left palp, apical view. C. $q$ scapus, lateral view. D. $q$ epigyne scapus, ventral view. E. + , spermathecae. Scale lines $=0.5 \mathrm{~mm}$. 
CARAPACE. As in male, but with more pronounced orange tinge.

Aвdomen. As in male, but more uniform grey; shoulders white; venter sepia with white stains; spinnerets orange brown.

EYes. MOQ: PME $=0.88$ AME. $\mathrm{Cl}=0.77$ DAME.

LEGS. Measurements:

I (13.26): Fe 4.12, Pa 1.57, Ti 3.18, Mt 3.06, Ta 1.33

II (11.18): Fe 3.25, Pa 1.45, Ti 2.55, Mt 2.20, Ta 1.73

III (6.81): Fe 2.31, Pa 1.02, Ti 1.29, Mt 1.33, Ta 0.86

IV (10.32): Fe 5.57, Pa 1.37, Ti 2.16, Mt 2.24, Ta 1.98

Epigyne (Fig. 3C-E). Length of scapus 0.47, diameter (lateral view) 0.16, L/D = 4.8. Spermathecae separated; scapus broader at tip (in ventral view), straight, evenly broad (in lateral view).

\section{Variation}

The males in our samples vary in length between 4.6 and $6.9 \mathrm{~mm}$, the females between 5.1 and $8 \mathrm{~mm}$.

The colour variation of the abdomen is comparable to that of the previous species.

\section{Distribution}

E. orientalis sp. nov. has an eastern distribution within the archipelago (Fig. 7): Española (arid zone), Islote Gardner near Española, Genovesa (arid zone), San Cristóbal (coastal arid zone and above $500 \mathrm{~m}$ alt.) and Santa Fé. We cannot detect a preference for a certain kind of vegetation zone, as most islands are low in altitude.

Eustala meridionalis sp. nov. urn:1sid:zoobank.org:act:AAB212B1-16FA-4110-8133-7BB2EEE7FB52

Figs 4 A-C, 5 B, 6 B, 7

Eustala vegeta (Keyserling, 1865) - Baert, Maelfait, Hendrickx \& Desender 2008: 47, map 15 (misidentification).

Eustala sp. - Baert 2013: 176, 178.

\section{Diagnosis}

Male: Differing from E. occidentalis and E. orientalis in the structure of the terminal apophysis, which is flat and broadening towards tip, apical ridge being slightly longer than caudal ridge. The female differs from both species by having a shorter and thicker scapus.

\section{Etymology}

The species name refers to its distribution in the southern part of the archipelago.

\section{Type material}

\section{Holotype}

o, ISLA FLOREANA, Cerro Pajas at the edge of Scalesia forest, alt. 335 m, 18 Apr. 1996 (P.96/55), leg. S. Peck. 
BAERT L., Three new Eustala (Araneae) species from the Galápagos Island

Allotype

+ , ISLA FLOREANA, SE slope of Cerro Pajas, Scalesia pedunculata with undergrowth of Tournefortia rufo-sericea and Croton scouleri, alt. 360 m, 20 Feb. 1977, leg. W.G. Reeder.

\section{Paratypes}

ISLA FLOREANA : 3 ふึึ, 1 (q), SE slope of Cerro Pajas, alt. 360 m, 20 Feb. 1977 (leg. W.G. Reeder); 1 đ̃, Base of Cerro Pajas, Scalesia forest, alt. 300 m, 16 Apr. 1996 (P.96/62) (leg. S. Peck); 1 jo, along road towards top of the island, transition to Scalesia woodland, vegetation mainly consisting of Tournefortia rufo-sericea and Scalesia pedunculata, thin shrub undergrowth, alt. 300 m, 22 Feb. 1988 (B.88/332) (leg. Baert, Maelfait \& Desender).

RBINS inventory number of all type material is I.G. 32723.

\section{Description}

Male (holotype)

LENGTH. Total length 5.02; carapace length 2.71, width 2.55, height 0.75 ; abdomen length 3.14 , width 2.39 , height 1.96 .

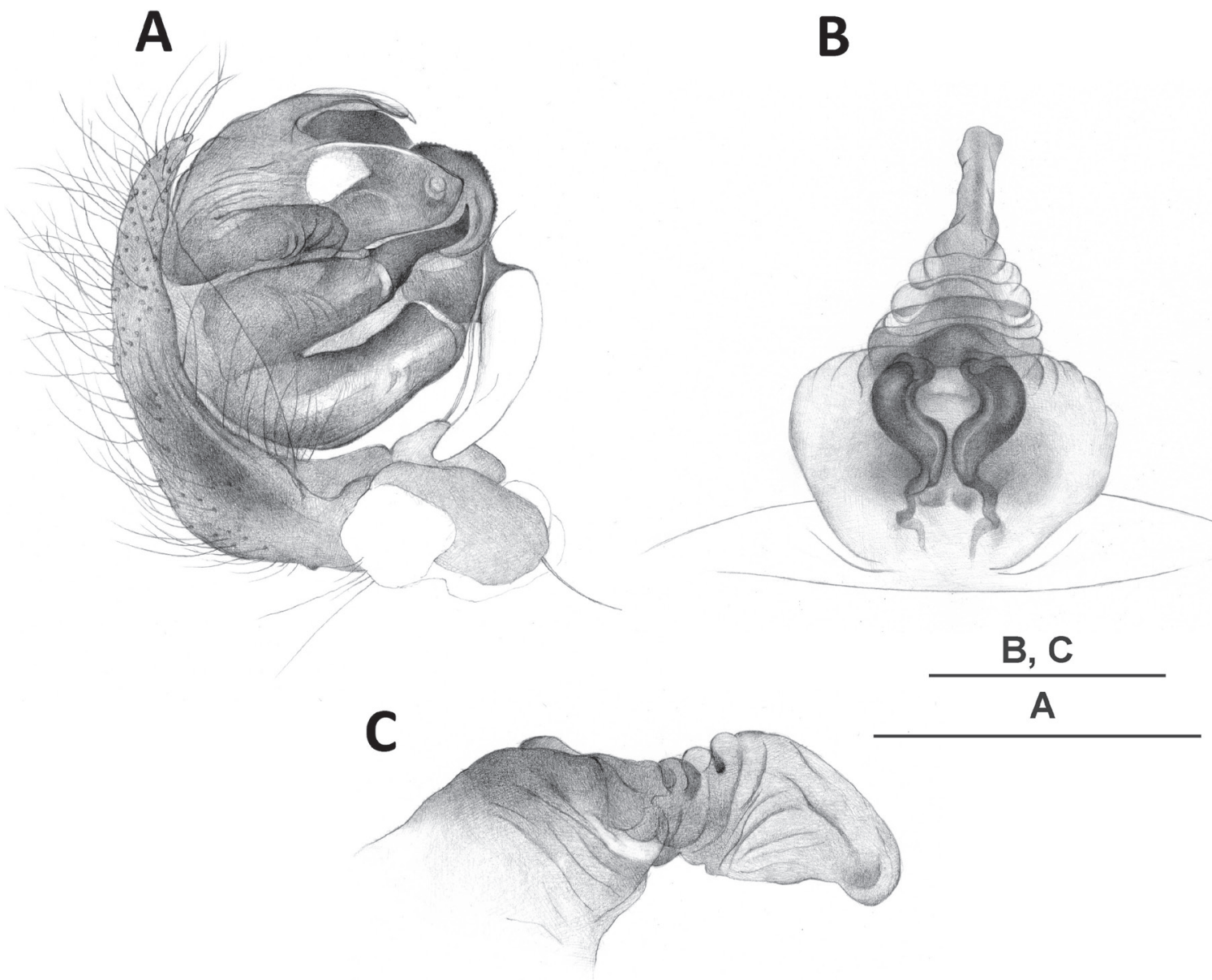

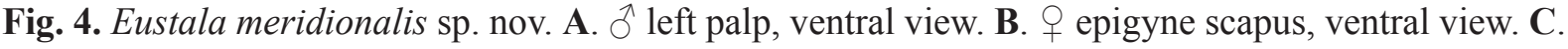
† scapus, lateral view. Scale lines $=0.5 \mathrm{~mm}$. 


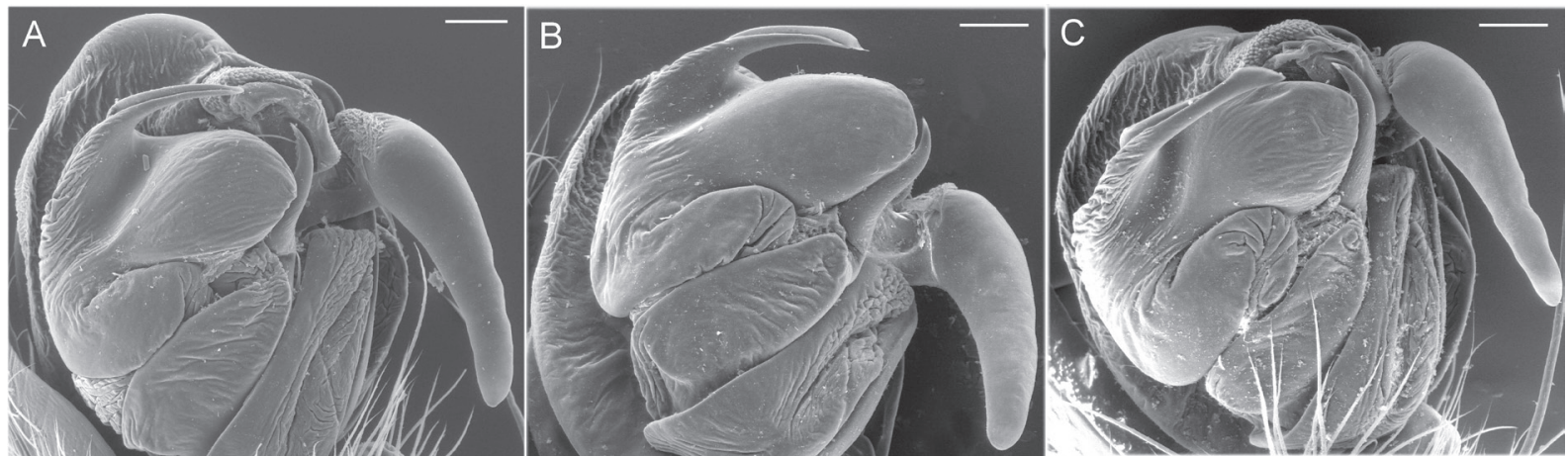

Fig. 5. Electron microscopic photographs of $\widehat{\jmath}$ left palp. A. Eustala occidentalis sp. nov. B. Eustala meridionalis sp. nov. C. Eustala orientalis sp. nov. Scale lines $=0.1 \mathrm{~mm}$.

CARAPACE. Colour (in alcohol) yellowish with black suffused striae and marked yellow V in cephalic region; two short combs of long, white hairs diverge from between PME and PLE. Chelicerae black with yellowish inner sides. Labium black with white apical edge. Endites black with white apical and inner margins. Sternum light yellow with broad, blackish suffused border. Pedipalps dark. Legs pale yellow with black annulations, variable among specimens; coxae and proximal part of femora whitish.

ABDOMEN. Dorsum greyish, speckled with cream spots; area between the converging waved brown lines darker grey. Venter light yellow, suffused with grey. Colulus and spinnerets black.

EYES. MOQ: PME $=0.73$ AME. $\mathrm{Cl}=0.63$ DAME.

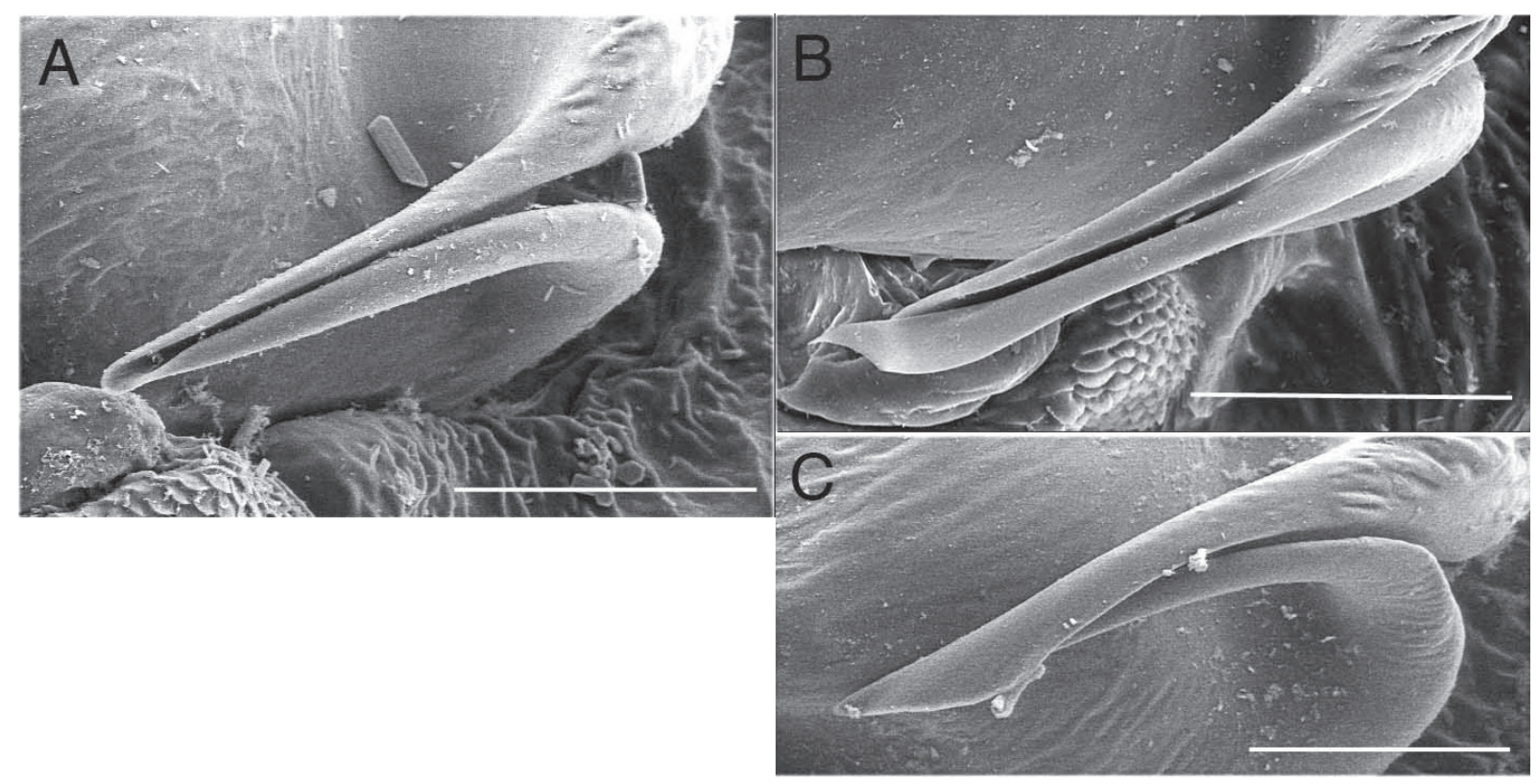

Fig. 6. Electron microscopic photographs of terminal apophysis of $\widehat{\jmath}$ left palp. A. Eustala occidentalis sp. nov. B. Eustala meridionalis sp. nov. C. Eustala orientalis sp. nov. Scale lines $=0.1 \mathrm{~mm}$. 
Legs. Measurements:

I (13.25): Fe 4.04, Pa 1.37, Ti 3.33, Mt 3.22, Ta 1.29

II (10.29): Fe 3.14, Pa 1.18, Ti 2.24, Mt 2.63, Ta 1.10

III (6.05): Fe 2.16, Pa 0.82, Ti 1.18, Mt 1.22, Ta 0.67

IV (9.22): Fe 3.14, Pa 1.14, Ti 1.96, Mt 2.16, Ta 0.82

Spination. All legs with numerous spines.

Pedipalp (Figs 4A, 5B). One very long patellar seta, a bit shorter than bulbus. Cymbium with conspicious, rounded, T-like tarsal hook. Terminal apophysis (Fig. 6B) flat and broadening towards tip, apical ridge of terminal apophysis being slightly longer than caudal ridge.

Female (allotype)

LENGTH. Total length 8.49; carapace length 3.53, width 2.86, height 1.37; abdomen length 5.18, width 4.90 , height 3.76 .

CARAPACE. Cream with some faint brown stains in cephalic area; eyes with black rings. Chelicerae cream. Labium yellow brown with white apical edge. Endites yellow brown with white apical and inner

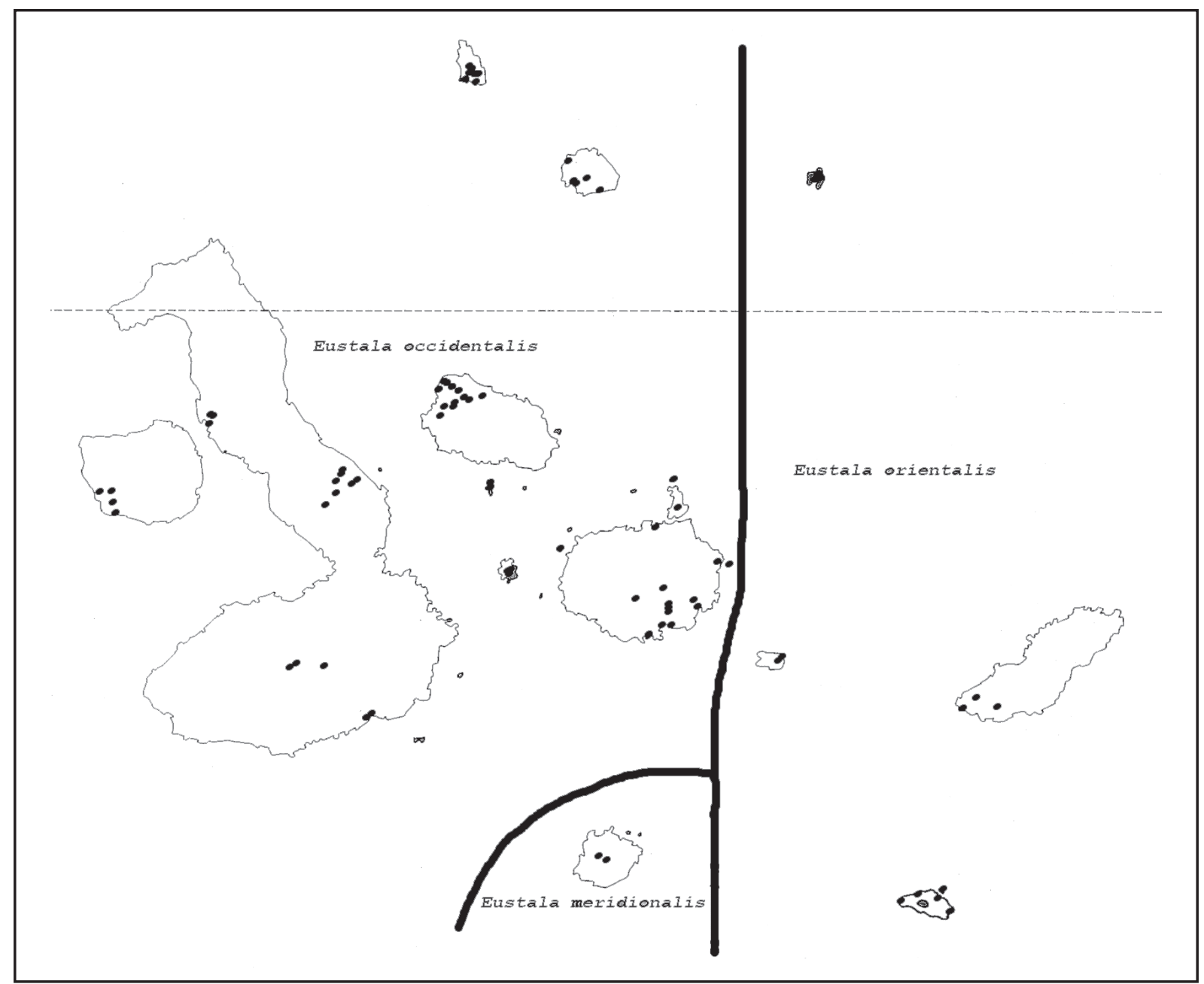

Fig. 7. Distribution map of Eustala occidentalis sp. nov., Eustala occidentalis sp. nov. and Eustala meridionalis sp. nov. 
edges. Sternum whitish with broad, yellowish brown border. Legs yellowish brown with annulations fainter than in male.

AвDomen. Dorsum lighter than in male; venter cream with three white stains. Colulus and spinnerets black with orange tinge.

EYEs. MOQ: PME $=0.92$ AME. $\mathrm{Cl}=0.80$ DAME.

LEGS. Measurements:

I (14.66): Fe 4.43, Pa 1.76, Ti 3.45, Mt 3.57, Ta 1.45

II (12.32): Fe 3.65, Pa 1.65, Ti 2.75, Mt 2.94, Ta 1.33

III (7.84): Fe 2.51, Pa 1.49, Ti 1.53, Mt 1.49, Ta 0.82

IV (10.98): Fe 3.69, Pa 1.53, Ti 2.35, Mt 2.43, Ta 0.98

Epigyne (Fig. 4 B-C). Scapus $0.35 \mathrm{~mm}$ long, $0.21 \mathrm{~mm}$ thick, L/D = 1.67. Spermathecae separated; scapus broader at tip (in ventral view), straight and very broad in the middle (in lateral view).

\section{Variation}

The few males in our samples vary between 4.70 and $5.90 \mathrm{~mm}$ in length. The male caught at the base of Cerro Pajas (P.96/62) shows the same two broad white spots as some E. occidentalis sp. nov. specimens (see colour pattern $\mathrm{C}$ of $E$. occidentalis sp. nov., Fig. $2 \mathrm{C}$ ). The other males all have a much lighter appearance. Their abdomen has more white and their cephalothorax is as light as that of the female allotype.

\section{Distribution}

E. meridionalis sp. nov. has only been found above $300 \mathrm{~m}$ alt. in the Scalesia zone of Cerro Pajas and its vicinity on the centrally located southern island Floreana (Fig. 7).

\section{Discussion}

The three Eustala species described here differ consistently from all mainland species: the males by the shape of the palpal terminal apophysis, the females by the shape of the epigyne scapus and the configuration of the spermathecae.

Chickering (1955) previously wrote that the colour pattern of the Central American Eustala species is highly variable. He therefore did not attribute much importance to this character in his species descriptions. This observation was later corroborated by Levi (1977: 96) in his generic diagnosis of Eustala: "Most species are variable in colouration with dark and light individuals, but most have a folium pattern on the dorsum, exceptions being some specimens of E. anastera that are contrastingly coloured with black patches on white in alcohol (figs. 219, 222)". The colour patterns as given here by Levi coincide strikingly with the patterns observed in Eustala species from Galápagos (Fig. 2, patterns A-B and E).

The three species show a very distinctive segregated distribution over the archipelago. E. occidentalis sp. nov. has the broadest range over the central (Santa Cruz), the western (Santiago, Isabela and Fernadina) and two of the northern islands (Pinta and Marchena). This part of the archipelago is younger than 2.3 my (Geist et al. 2013). E. meridionalis sp. nov. lives on the isolated southern island of Floreana which has an age between 1.5 and 2.3 my (Geist et al. 2013). E. orientalis sp. nov. shows a peculiar distribution which I cannot explain. Its presence on the eastern islands San Cristóbal and Española may be logical and understandable, these islands being the oldest ones (between 2.4 and $4.0 \mathrm{My}$, Geist et al. 2013). But its presence on the "eastern" islands Santa Fé and Genovesa is less explicable, since 
BAERT L., Three new Eustala (Araneae) species from the Galápagos Island

the distance separating Santa Fé and Santa Cruz, where E. occidentalis sp. nov. lives, hardly exceeds $17.5 \mathrm{~km}$. Moreover, Genovesa belongs to the very young northern islands (age between 0.3 and 0.8 my according to Geist et al. 2013), lying on a straight line north of Santa Fé along the $90^{\circ} \mathrm{W}$ longitude, at a distance of approximately $125 \mathrm{~km}$, but only some $50 \mathrm{~km}$ east of the central northern island Marchena. Odo desenderi Baert, 2009 has a similar distribution. It occurs on Santa Fé and Genovesa but in an inexplicable way also on Marchena.

Eustala species are, like most araneids, probably good ballooners, which can easily be transported by wind. One should expect $E$. orientalis sp. nov. to be able to bridge the short northwest distance between Santa Fé and Santa Cruz, and to be found on that central island. This has apparently not happened, although the prevailing winds come from the southeast.

The distribution of E. orientalis sp. nov. on the older southeastern islands and E. occidentalis sp. nov. on the younger central and northwestern islands fits the line of the progression rule pattern, paralleling the geological formation of the islands (Wagner \& Funk 1995; Parent et al. 2008).

\section{Natural History}

Eustala species are apparently nocturnal (Levi 1977: 97) and remove their web during daytime. They seem to have no retreat, but rest on a branch of the vegetation in which they live (Levi 1977: 97).

They were mostly caught by sweeping in daytime and were sometimes caught at night.

\section{Acknowledgements}

Field assistance has been given by Jean-Pierre Maelfait $\uparrow$ (1982-2002), Konjev Desender $\uparrow$ (1982-2000), Frederik Hendrickx $(2002,2010)$ and Wouter Dekoninck $(2009,2010)$ (Belgium); Stewart Peck \& John Heraty (Canada); Heinrich \& Irene Schatz (Austria); Sandra Abedrabbo, Sonja Sandovall, Germania Estevez, Lazaro Roque and Henri Herrera (Ecuador).

We received logistic and technical support from the Charles Darwin Research Station (CDRS) and the Parque Nacional de Galápagos (SPNG). Financial support was provided by the Belgian State (now Belspo), FWO and the Léopold III Fund.

Thanks go to James Reddell of the Texas Memorial Museum (University of Texas) for the loan of the spider collections made by W.G. Reeder, to Janet Beccaloni of the Natural History Museum of London for the loan of the type specimens of Eustala vegeta (Keyserling, 1865) and to Laura Leibensperger of the Harvard University Museum of Comparative Zoology for the loan of various Eustala species.

Special thanks go to Marylise Leclercq who made all the drawings. I further thank Julien Cillis who made the SEM-photographs and Alain Pauly who made the colour photographs.

This publication is contribution number 2091 of the Charles Darwin Foundation for the Galápagos Islands.

\section{References}

Baert L. 2013. Summary of our present knowledge of the spider communities of the Galápagos archipelago. First analysis of the spider communities of the islands Santa Cruz and Isabela. Belgian Journal of Zoology 143 (Supplement): 159-185.

Baert L., Maelfait J.-P., Hendrickx F. \& Desender K. 2008. Distribution and habitat preference of the spiders (Araneae) of Galápagos. Bulletin van het Koninklijk Belgisch Instituut voor Natuurwetenschappen, Entomologie 78: 39-111. 
Banks N. 1902. Papers from the Hopkins Stanford Galápagos Expedition. 1898-1899. VII. Entomological results (6), Arachnida. Proceedings of the Washington Academy of Sciences 4: 49-70. http://www.biodiversitylibrary.org/item/35272\#page/65/mode/1up

Banks N. 1924. Arachnida of the Williams Galápagos Expedition. Zoologia V (9): 93-99.

Chickering A.M. 1955. The genus Eustala (Araneae, Argiopidae) in Central America. Bulletin of the Museum of Comparative Zoology 112(6): 391-518. http://www.biodiversitylibrary.org/ item/21255\#page/423/mode/1up

Garrett S.E., Conner W.E. \& Roque-Albelo L. 2008. Alkaloidal protection of Utetheisa galapagensis (Lepidoptera: Arctiidae) against an invertebrate and a vertebrate predator in the Galápagos Islands. Galápagos Research 65: 2-6.

Geist D., Snell H., Snell H., Goddard C. \& Kurz M. 2013. A paleogeographic model of the Galápagos Islands and biogeographical and evolutionary implications. In: Harp K.S., Mittelstaedt E., d'Ozouville N. \& Graham D.W. (eds) The Galápagos: A Natural Laboratory for the Earth Sciences, American Geophysical Union Monograph. American Geophysical Union, Washington DC.

Jocqué R., Alderweireldt M. \& Dippenaar-Schoeman A. 2013. Biodiversity, an African perspective. In: Penny D. (ed.) Spider Research in the 21 $1^{\text {st }}$ Century: 18-57. Siri Scientific Press, Manchester.

Levi H.W. 1977. The American orb-weaver genera Cyclosa, Metazygia and Eustala north of Mexico (Araneae, Araneidae). Bulletin of the Museum of Comparative Zoology 148 (3): 61-127. http://www. biodiversitylibrary.org/page/4314711\#page/79/mode/1up

Parent C.E., Caccone A. \& Petren K. 2008. Colonization and diversification of Galápagos terrestrial fauna : a phylogenetic and biogeographical synthesis. Philosophical Transactions of the Royal Society B, 363: 3347-3361. http://dx.doi.org/10.1098/rstb.2008.0118

Platnick N. 2014. The World Spider Catalogue. Version 14.5. American Museum of Natural History, Washington DC, USA. http://dx.doi.org/10.5531/db.iz.0001

Roth V.D. \& Craig P.R. 1970. Arachnida of the Galapagos Islands (excluding Acarina). In: Leleup N. \& Leleup J. (eds) Mission zoologique belge aux Iles Galapagos et en Ecuador. Résultats scientifiques, deuxième partie: 107-124. Musée Royal de l'Afrique Centrale, Tervuren.

Snodgrass R.E. 1902. Papers from the Hopkins Stanford Galápagos Expedition. 1898-1899. VII. Entomological results (6), Arachnida. Part II. Field notes. Proceedings of the Washington Academy of Sciences 4: 71-80. http://www.biodiversitylibrary.org/item/35272\#page/87/mode/1up

Wagner W.L. \& Funk V.A. 1995. Hawaiian Biogeography. Smithsonian Institution, Washington DC.

Manuscript received: 16 January 2014

Manuscript accepted: 24 April 2014

Published on: 5 June 2014

Topic editor: Rudy Jocqué

Desk editor: Kristiaan Hoedemakers

Printed versions of all papers are also deposited in the libraries of the institutes that are members of the EJT consortium: Muséum National d'Histoire Naturelle, Paris, France; Botanic Garden Meise, Belgium; Royal Museum for Central Africa, Tervuren, Belgium; Natural History Museum, London, United Kingdom; Royal Belgian Institute of Natural Sciences, Brussels, Belgium; Natural History Museum of Denmark, Copenhagen, Denmark. 